\title{
Evaluation of predictors of postoperative complications of major surgery programs in patients with type 2 diabetes. P-0617
}

\author{
Authors: J.N. Giunta1 ${ }^{1}$, M.M. Castex¹, A.M. Alvarez', L.I. Capurroํ, C.M. Elizondo², F.C. Bonofiglio³, M.F. \\ Russo Picasso ${ }^{1}$, L.E. Litwak ${ }^{1}$, P. Fainstein Day ${ }^{1}$, D.H. Giunta ${ }^{2}$, L.A. Grosembacher ${ }^{1}$. \\ ${ }^{1}$ Hospital Italiano de Buenos Aires, Endocrinology, CABA, Argentina. \\ ${ }^{2}$ Hospital Italiano de Buenos Aires, Department of Clinical Research, Caba, Argentina. \\ ${ }^{3}$ Hospital Italiano de Buenos Aires, Anesthesiology, Caba, Argentina.
}

\section{Background}

Diabetes and its complications are highly prevalent in the preoperative evaluation of programmed surgeries. Diabetic patients have more immediate postoperative complications and more long-term complications in both cardiological and noncardiological programmed surgeries. The most frequent complications are infections, cardiovascular events and death. Patients with a history of uncontrolled glycemia represent the fraction of the population that has more elevated values of $\mathrm{HbA1c}$. It is not known if these patients are at greater risk of postoperative complications after the acute stress of programmed surgery.

\section{Objective}

To evaluate the association between $\mathrm{HbA} 1 \mathrm{c}$ and postoperative complications of programmed major surgeries in patients with type 2 diabetes mellitus.

\section{Methods}

This is a cohort study. It was conducted to measure $\mathrm{HbA1c}$ in adult patients with a diagnosis of type 2 diabetes who require programmed major surgery. The primary endpoints were Infections, Admissions, Cardiovascular Events, Cerebrovascular Events, Death and Composite Endpoint.

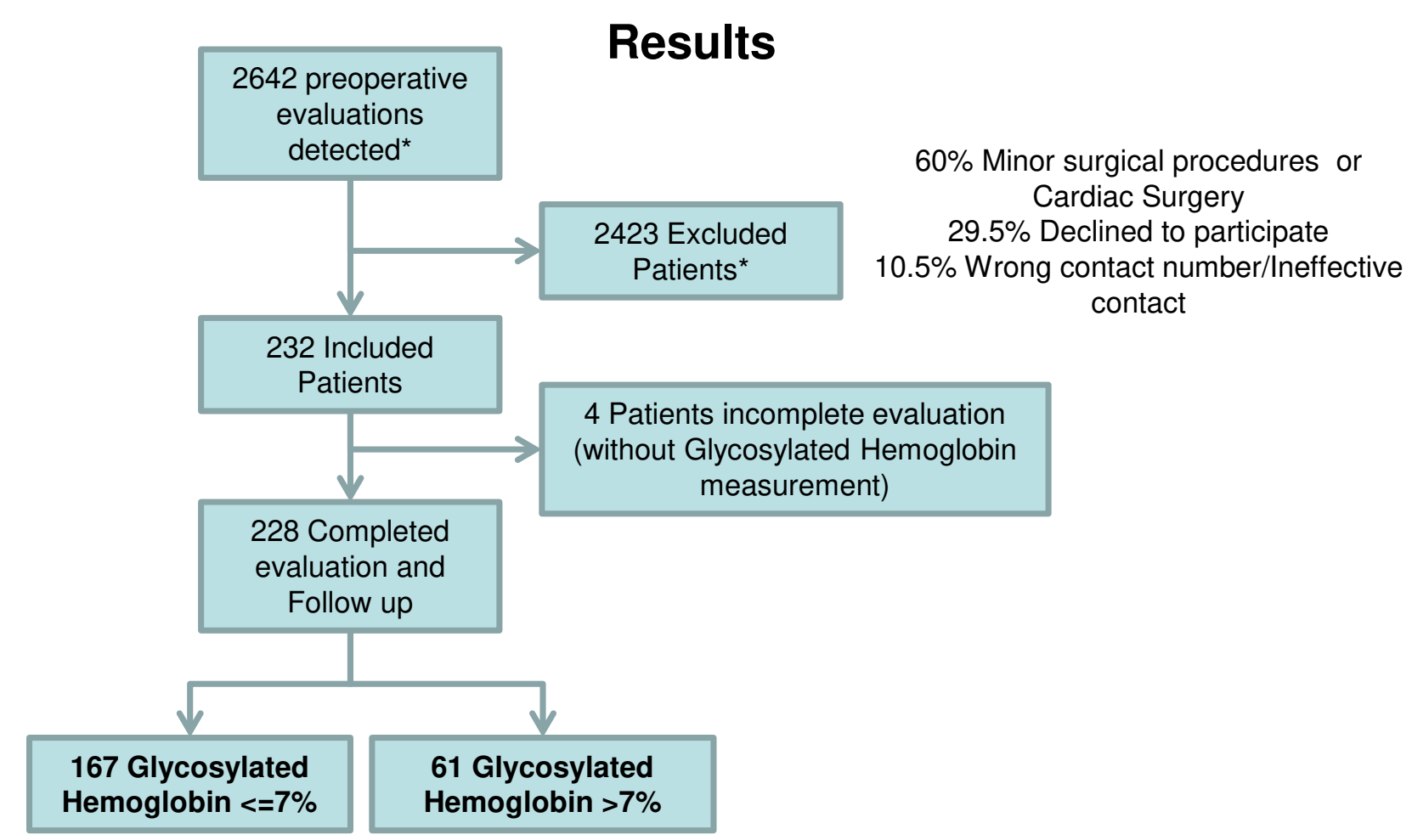

\begin{tabular}{|l|c|c|c|}
\hline $\begin{array}{l}\text { Baseline } \\
\text { Characteristics }\end{array}$ & $\begin{array}{c}\text { Glycosylated } \\
\text { Hemoglobin <=7\% (167) }\end{array}$ & $\begin{array}{c}\text { Glycosylated } \\
\text { Hemoglobin >7\% (61) }\end{array}$ & $\mathbf{P}$ \\
\hline Age (years) & $\mathbf{1}$ & $63.44(12.7)$ & 0.126 \\
\hline Masculine Gender & $50.48(9.5)$ & $62.3 \%(38)$ & 0.192 \\
\hline BMI $^{2}$ & $30.9(7.7)$ & $31.53(8)$ & 0.523 \\
\hline Smoking Status & $12.65(21)$ & $11.48(7)$ & 0.81 \\
\hline Hypertension & $85.03(142)$ & $78.69(48)$ & 0.25 \\
\hline Dyslipidemia & $64.07(107)$ & $54.10(33)$ & 0.17 \\
\hline Coronary Heart Disease & $25.9(43)$ & $16.39(10)$ & 0.13 \\
\hline Cancer & $35.33(59)$ & $37.7(23)$ & 0.74 \\
\hline $\begin{array}{l}\text { Time since Diabetes } \\
\text { diagnosis (years) }\end{array}$ & $5.5(8)$ & $9.5(11)$ & 0.005 \\
\hline Diabetic Nephropathy & $7.83(13)$ & $11.48(7)$ & 0.39 \\
\hline $\begin{array}{l}\text { Diabetic Retinopathy } \\
\text { Hospitalization time } \\
\text { (days) }\end{array}$ & $\mathbf{1 . 9 2 ( 3 )}$ & $\mathbf{1 0 . 7 1 ( 3 )}$ & $\mathbf{0 . 0 1 2}$ \\
\hline $\begin{array}{l}\text { Glycosylated } \\
\text { Hemoglobin (\%) }\end{array}$ & $3(3)$ & $3(2)$ & 0.930 \\
\hline $\begin{array}{l}\text { 1 mean (standard deviation, sd); 2 median (inter-quartile range, iqr); 3 Mann-Whitney U } \\
\text { test }\end{array}$ & & & \\
\hline
\end{tabular}

Moss, S.E., R. Klein, and B.E. Klein, Risk factors for hospitalization in people with diabetes. Arch Intern Med, 1999. 159(17): p. 2053-7. Krolikowska, M., et al., Mortality in diabetic patients undergoing non-cardiac surgery: a 7-year follow-up study. Acta Anaesthesiol Scand, 2009. Juul, A.B., J. Wetterslev, and A. Kofoed-Enevoldsen, Long-term postoperative mortality in diabetic patients undergoing major non-cardiac surgery. Eur J Anaesthesiol, 2004. 21(7): p. 523-9.

Subramaniam, B., et al., Continuous perioperative insulin infusion decreases major cardiovascular events in patients undergoing vascular surgery: a prospective, randomized trial. Anesthesiology, 2009. 110(5): p. 970-7.

\begin{tabular}{|l|c|c|c|c|}
\hline $\begin{array}{l}\text { Association between } \\
\text { Glycosylated } \\
\text { Hemoglobin and } \\
\text { Endpoints }\end{array}$ & HbA1c >7\% & HbA1c <=7\% & HR & P \\
\hline Infection \%(n) & $8.98(15)$ & $16.39(10)$ & $\begin{array}{c}0.92(0.68- \\
1.23)\end{array}$ & p 0.599 \\
\hline Re-admission \%(n) & $7.7(13)$ & $13.11(8)$ & $\begin{array}{c}1.59(0.66- \\
3.85)\end{array}$ & $p 0.296$ \\
\hline $\begin{array}{l}\text { Cardiovascular Event } \\
\%(n)\end{array}$ & -- & $6.56(4)$ & -- & -- \\
\hline $\begin{array}{l}\text { Cerebrovascular } \\
\text { Events \%(n) }\end{array}$ & $0.6(1)$ & -- & -- & -- \\
\hline Death \%(n) & -- & $3.28(2)$ & -- & -- \\
\hline $\begin{array}{l}\text { Composite } \\
\text { Endpoint\%(n) }\end{array}$ & $\mathbf{1 3 . 7 ( 2 3 )}$ & $\mathbf{2 7 . 8 ( 1 7 )}$ & $\begin{array}{c}\mathbf{2 . 0 5}(\mathbf{1 . 0 9} \\
\mathbf{3 . 8 4}\end{array}$ & $\mathbf{p ~ 0 . 0 2 4}$ \\
\hline
\end{tabular}

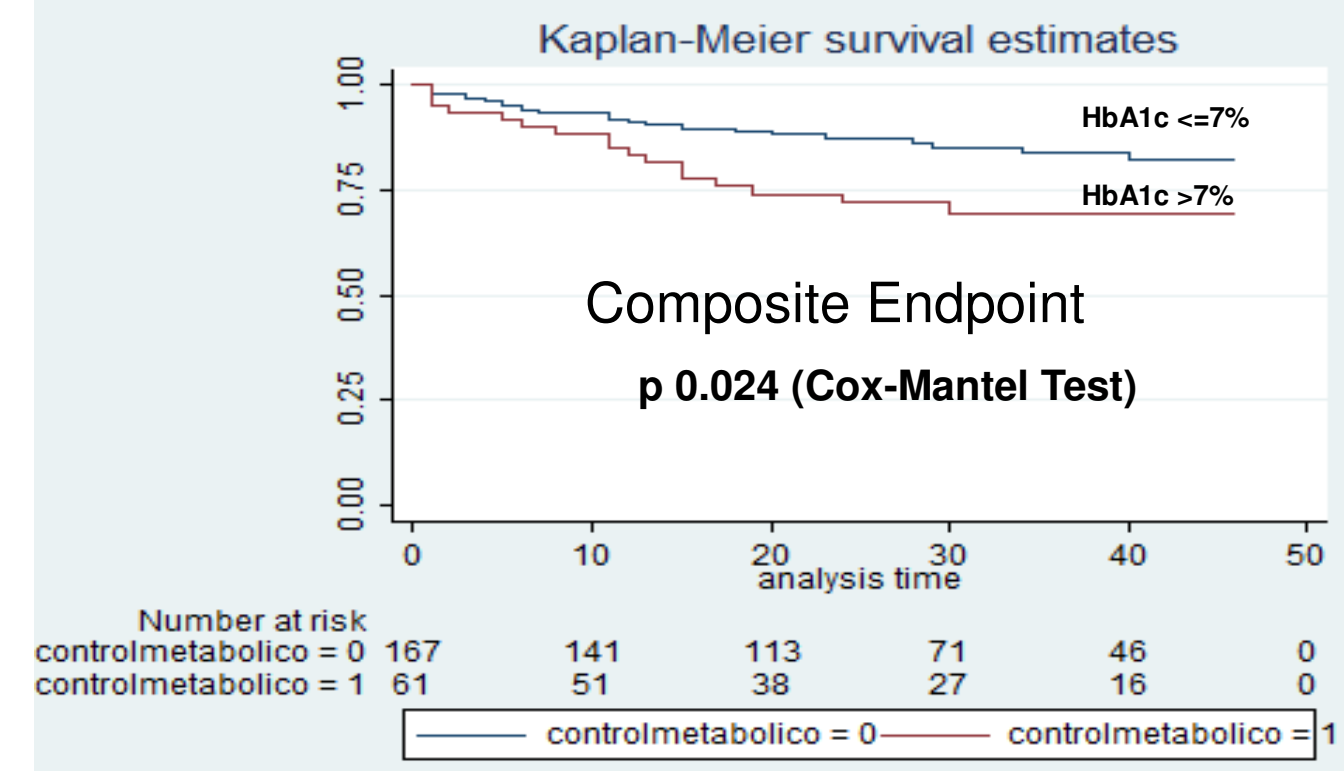

\begin{tabular}{|l|c|}
\hline $\begin{array}{l}\text { Association between baseline } \\
\text { characteristic and Endpoints }\end{array}$ & $\begin{array}{c}\text { Hazard Ratio for Composite } \\
\text { Endpoint (40) }\end{array}$ \\
\hline $\begin{array}{l}\text { Metabolic control evaluated with } \\
\text { HbA1c (crude HR) }\end{array}$ & $2.05(1.09-3.84)$ p 0.024 \\
\hline Age (years) & $1.04(1.008-1.07)$ p 0.013 \\
\hline Diabetic Nephropathy & $2.92(1.29-6.63)$ p 0.010 \\
\hline $\begin{array}{l}\text { Diabetic Retinopathy } \\
\begin{array}{l}\text { Metabolic Control Adjusted for } \\
\text { age, Retinopathy and } \\
\text { Nephropathy }\end{array}\end{array}$ & $4.35(1.69-11.18)$ p 0.002 \\
\hline
\end{tabular}

Discussion

This study identified as predictors of postoperative complications, the glycemic control measured by HbA1c, the age of the patient, the presence of nephropathy and retinopathy for combined event. Therefore, these predictors prior to major surgery programs in patients with type 2 diabetes establishes an increase in postoperative complications.

This research do not have conflicts of Interest. This poster does not contain any trade names. This poster does not cover any unapproved uses of specific drugs, other products or devices. 